\title{
Corrientes antropo-filosóficas y su implicación en el currículo de la educación física y afines en Colombia
}

\author{
Anthropological and philosophical trends: their impact on physical education and alike curriculum in Colombia
}

\author{
Simón Cruz Fajardo*
}

\section{Resumen}

Esta es una investigación de enfoque cualitativo y cuantitativo. Es un trabajo de tipo documental; y por tanto, recurre al análisis de contenido. Aborda el problema de cuáles han sido las relaciones entre las principales concepciones antropo-filosóficas universales y las propuestas curriculares de la educación física. Con base en ello, analiza algunos de los programas vigentes en el contexto colombiano para establecer la perspectiva antropología filosófica desde la cual emanan. El objetivo es describir el pensamiento filosófico de las más representativas corrientes antropológicas en la historia universal, analizando sus contenidos e infiriendo sus influencias en los respectivos currículos educativos; y además, sugerir algunas ideas alternativas para el diseño de un currículo unitario para la educación física. Se concluye que los currículos de la educación física y la cultura física, se sustentan aún en el dualismo antropológico platónico-cartesiano y la antropología monista científicista, y que es posible una alternativa curricular que favorezca una reflexión unicista del hombre como unidad psico-orgánico-conductual.

\section{Abstract}

Antropología filosófica, hombre, dualismo, monismo, unicismo, educación física, cultura física, currículo.

This research is based on a qualitative-quantitative approach. It is a documentary work; so, it analyses the content. It examines which have been the relations among principal anthropological-philosophical theories and positions related to physical education curricula. Some programs in force in the Colombian context are examined for determining what anthropological-philosophical point of view they come from. This article aims to describe the most important anthropological theories analysing their contents and inferring their influences on several educational curricula. Besides, it suggests some alternative ideas for design a unitary curriculum for Physical Education. This work concludes that Physical Education curricula and physical culture are still supported in the anthropological Platonist-Cartesian dualism and monist-scientist Anthropology, as well as an alternative curriculum in favour of a reflexion on human being as a psycho-organic-behavioural unit is possible.

Key words: Philosophical anthropology, human being, dualism, monism, unicism, physical education, physical culture, curriculum.

Fecha de recepción: 5 de octubre de 2007.

Fecha de aceptación: 2 de noviembre de 2007.

\footnotetext{
* Licenciado en Educación Física, Universidad Pedagógica Nacional. Especialista en Pedagogía y Didáctica de la Educación Física, Universidad Pedagógica Nacional. Especialista en Pedagogía del Folclor, Universidad Santo Tomás. Magíster en Filosofia Latinoamericana, Universidad Santo Tomás. Estudios teológicos en proceso.
} 


\section{Introducción}

Dentro de la formación humanista que imparte la Universidad Santo Tomás en todas sus carreras, la Antropología Filosófica ocupa un puesto de especial importancia. El hombre, en definitiva, constituye el objetivo último de todas las ciencias. Cuando la formación profesional olvida este objetivo terminal, resulta fácil explicar el por qué de tanto reduccionismo a lo puramente técnico ${ }^{1}$.

Eudoro Rodríguez

Hoy más que nunca es evidente lo que ya sospechaba el filósofo español Ortega y Gasset respecto a la tendencia occidental hacia la reivindicación de su cuerpo, a una resurrección de la carne, y lo verificado posteriormente por su colega y compatriota Pedro Laín Entralgo, al afirmar la patenticidad en la vida actual de la pasión por el cuerpo, dado el auge práctico y contemplativo del deporte, del excesivo cuidado de la salud y la invasión del nutricionismo dietético y la cosmetología. El campo que nos ocupa es efectivamente el deporte y su explícita inclusión en el currículo de la educación física escolar y como formación profesional universitaria, pero desde la antropofilosofía.

Se consideran cuatro premisas problémicas: a) la subvaloración profesional de la educación física y la cultura física por su reduccionismo tecnológico; b) la influencia del concepto filosófico sobre el hombre en lo que él hace consigo mismo y con otros; c) la implícita intencionalidad que toda pedagogía tiene en la formación humana y social; y d) el objetivo último de la formación profesional es el hombre; por tanto, la antropología filosófica es necesaria en ella. Desde allí, nos preguntamos por las causas de dicha subvaloración, los postulados de las corrientes antropofilosóficas, su relación con las propuestas educativas respectivas y la perspectiva curricular actual para la cultura física y la educación física.

Entre nuestras razones están un esfuerzo por el reconocimiento disciplinar en la comunidad académica y científica, la necesidad de una fundamentación antropofilosófica, la claridad en la intención pedagógica y la autorreflexión crítica. Los alcances obtenidos fueron: la descripción general de las principales corrientes antropofilosóficas, la relación del pensamiento filosófico de éstas con los respectivos proyectos educativos de la educación física y el aporte de una reflexión antropofilosófica actual para el currículo de la educación física y cultura física.

1 Rodríguez A., Eudoro y otros. Antropología: perspectiva latinoamericana. Bogotá, Universidad Santo Tomás de Aquino, 1996, p. XI.
El marco teórico y temático considera las tres principales antropologías filosóficas reconocidas en la historia: la concepción antropofilosófica dualista ${ }^{2}$; la antropofilosofía monista ${ }^{3}$, especialmente la versión materialista; la mirada antropofilosófica uinicista ${ }^{4}$. Además, se consideran algunas pautas hacia una aproximación curricular para una cultura física más integral, humanista y unitaria. La metodología utilizada fue de tipo descriptiva en las diferentes corrientes antropofilosóficas con un enfoque cualitativo, recurriendo a las estrategias investigativas documental, análisis de contenido y evaluativa. Ésta última tuvo un enfoque cuantitativo dado el análisis estadístico sobre los planes de estudio de algunos programas de cultura física y afines en Colombia.

\section{Problema}

La educación física y afines no han gozado - ni gozan aún- de un estatus social, académico y científico significativo, en comparación con otras profesiones como la medicina, las ingenierías, las ciencias económicas, el derecho y la misma filosofía entre otras. El currículo mantiene un énfasis en las ciencias naturales sin considerar la antropología humanista implícita en su propia naturaleza. El quehacer pedagógico se ha limitado a la formación técnica. Docentes y estudiantes no encuentran relaciones con el campo de las humanidades, entre ellas la antropología filosófica. En consecuencia, se ha fomentado una educación y cultura física alejadas de su responsabilidad humana total y su integralidad multiinter-transdisciplinar.

A pesar de que se observa algún adelanto en casos aislados -como este mismo trabajo-, nos preguntamos cuál es la causa de este ciclo vicioso que subvalora la educación física, la cultura física y afines. Sabiendo que del concepto filosófico sobre el hombre depende lo que este hace consigo mismo o con los demás, nos llevó a cuestionar cuáles han sido las concepciones antropofilosóficas en la historia del pensamiento y el lugar que en

\footnotetext{
2 Platón en la antigüiedad y Descartes en la modernidad son los principales promotores de la concepción antropológica dicotómica al asumir al hombre como una composición de dos elementos.

3 Las concepciones monistas consideran esencial uno de los dos elementos constitutivos del hombre: el alma o el cuerpo. Parménides en la antiguiedad y Spinoza en la modernidad son los principales defensores de la concepción antropológica espiritualista, mientras la ciencia se ocupa del materialismo del hombre.

4 El pensamiento semita y Aristóteles desde la antigüedad, San Agustín y Santo Tomás en el medioevo, Zubiri y Laín Entralgo son algunos de los pensadores de esta corriente antropofilosófica.
} 
ellas ocupa el cuerpo humano. Entendiendo que toda pedagogía conlleva una intencionalidad de formación humana, inquirimos sobre cuáles han sido las propuestas educativas curriculares en los grandes momentos históricos. Como consecuencia de ello, indagamos a cerca de qué influencias se pueden inferir en relación con las correspondientes antropologías filosóficas. Finalmente, buscamos cómo ha ser hoy el currículo de la educación física, cultura física y afines desde la antropología filosófica.

\section{Justificación}

La educación física y la cultura física son hechos de naturaleza antropológica y trascendencia social, como cualquier otro ámbito del saber; por tanto, requieren la pesquisa investigativa sobre sus fundamentos y su consecuente reconocimiento profesional. El humanismo promueve una formación integral no entendida como yuxtaposición de saberes sino como su convergencia en unidad, donde la antropología filosófica ocupa un lugar fundamental para superar el reduccionismo profesional a lo puramente técnico, pues considera que el hombre es su objetivo último hacia el bienestar de la nación y el perfeccionamiento de la humanidad. Por ello, se deben ocupar -además de la dimensión "física"- de las dimensiones intelectual, estética, ética, política y económica, entre otras. Así como la pedagogía como ciencia del hecho educativo exige a la educación física y ahora la cultura física sus principios pedagógicos y didácticos; la antropología filosófica les reclama sus fundamentos antropológicos filosóficos y epistemológicos.

\section{Objetivos}

\section{Generales}

Interpretar las principales corrientes antropofilosóficas propuestas a través de la historia.

Argumentar una antropología filosófica actual que fundamente el currículo de la educación física.

Proponer una alternativa actualizada del currículo de la educación física desde la antropología filosófica.

\section{Específicos}

Interpretar las concepciones antropofilosóficas dualistas (Platón y Descartes), monistas (Parménides, Spinoza y la Ciencia), y unicistas (Semitas, Aristóteles, San Agustín, Santo Tomás, Xavier Zubiri y Pedro Laín Entralgo).
- Argumentar las influencias de dichas antropofilosofías en las correspondientes propuestas curriculares de la educación física.

- Proponer algunas ideas reflexivas desde una perspectiva antropofilosófica unicista para el diseño de un currículo actual, integral-humanista de la educación física.

\section{Material}

El material simbólico de los textos alusivos al tema que se contemplan como marco teórico corresponde a las obras de las tres principales explicaciones del pensamiento filosófico acerca del hombre y una teoría integral y humanista del currículo.

El universo principal de las fuentes bibliográficas investigadas son las obras de Platón (República y Fedón), Descartes (Discurso del método, Meditaciones metafísicas), Parménides (Fragmentos), Spinoza (Ética demostrada según el orden geométrico), Aristóteles (Sobre el alma), San Agustín (La ciudad de Dios), Santo Tomás de Aquino (Suma teológica, Suma contra los gentiles y Del ente y la esencia), Xavier Zubiri (Sobre el hombre y Siete ensayos de antropología filosófica) y Pedro Laín Entralgo (El cuerpo humano: teoría actual, Qué es el hombre: evolución y sentido de la vida, y Cuerpo y alma: estructura dinámica del cuerpo humano). Además, se tienen en cuenta textos de Enrique Dussel (El humanismo semita), Fideligno Niño Meza (Estudio de currículo: hacia una reestructuración de currículo universitario en clave humanística), y Nelson E. López Jiménez (Reestructuración curricular de la educación superior: hacia la integración del saber y Modernización curricular de las instituciones educativas, los PEI de cara al siglo XXI).

\section{Metodología}

El diseño metodológico utilizado en esta investigación es cualitativo en el sentido de la observación, descripción histórica y análisis de contenido de las principales corrientes antropofilosóficas (dualismo, monismo y unicismo) en las obras de los filósofos más representativos, y la de la teoría curricular integral y humanista de autores reconocidos en el ámbito de la educación y la filosofía. Cuantitativamente, en el momento de la evaluación diagnóstica que se realizó sobre algunos programas de formación profesional en educación física y cultura física en pregrado en Colombia, especialmente sobre los planes de estudio clasificados por campos de disciplinas y saberes. 


\section{Resultados}

Luego de analizar las concepciones filosóficas sobre el hombre desde la perspectiva dualista, monista y unicista, relacionarlas con las propuestas curriculares correspondientes se han encontrado los siguientes resultados:

La antropología filosófica dualista de Platón concibió el hombre como un compuesto de dos elementos separables y un dualismo curricular:

El pensamiento antropológico platónico se basó en su "Teoría de los dos mundos" -el mundo de las ideas y el de los sentidos-. Desde allí afirmó que el hombre era un compuesto separable de dos elementos: el alma y el cuerpo, cuya unión se daba de manera meramente "accidental", tal como el cochero y la carroza. A su vez, al considerar la superioridad de ésta sobre éste dada la naturaleza espiritual e incorruptible de aquella frente a la material y corruptible de aquel (Platón, 2003: 565), defendió la importancia del alma como fuente de la verdad y despreció la utilidad el cuerpo para alcanzarla (Platón, 2003: 549). Del mismo modo, al clasificar el alma en racional (ideal para gobernar), concupiscible (ideal para la producción) e irascible (ideal para la fuerza), y referirla a los hombres para el servicio del Estado, incapacitó a artesanos, gladiadores y esclavos para pensar y decidir (Gajate, 1999: 11). El cuerpo humano es tan sólo como tumba y una cárcel para el alma (Platón, 2003: 270).

El currículo de la antigüedad platónica se planteó desde su perspectiva dualista. El mismo Platón estableció una paideia música (cultivo intelectual, estético y moral) junto a su otra parte paideia gimnasia (Jaeger, 1997: 624) (cultivo corporal), dos escuelas: la "escuela de música" donde recibía enseñanzas intelectuales y morales (Jaeger, 1997: 35) y la "palestra" donde se preparaba en gimnasia (Gajate, 1999: 15). Así, se hizo evidente una educación intelectual y una educación física. El ideal educativo platónico ${ }^{5}$ puede resumirse en la música para el alma, la gimnasia para el cuerpo y ambos para el Estado. A pesar de la primacía platónica del alma sobre el cuerpo y la consecuente importancia del espíritu mediante la música, el fortalecimiento físico connotó importancia para la formación del guerrero, un artista y un ciudadano (Gajate, 1999: 34) practicando la gimnasia desde la infancia (Jaeger, 1997: 624). Sin embargo, quienes participaban en los "Juegos Olímpicos" no eran precisamente ellos, sino los gladiadores y esclavos que

5 Curiosamente, Aristocles fue llamado Platón debido -según algunos- a que tenía la espalda ancha; de acuerdo a otros, por tener la frente grande. aspiraban a la libertad de la esclavitud grecorromana. su vez, consideró débiles a los que se dedicaban sólo a intelecto, y brutos a aquellos que se consagraban solamente a la gimnasia.

La antropología filosófica dualista de Descartes im plicó un dualismo curricular racionalista y su consecuente subvaloración extensiva de la educación física:

El pensamiento antropológico cartesiano se fundamentó en su "teoría de la duda metódica" (Descartes 2004a: XIII) mediante la cual planteó su desconfianza respecto de los sentidos para el conocimiento de la verdad, pues engañan. Con ese postulado afirmó que solamente el pensar determina el existir. De allí se sigue que el hombre es "una cosa que piensa" (Descartes, 2004b 67) y el cuerpo es una mera extensión (Descartes, 1977 25-26). Así concibe al hombre como un compuesto de dos realidades diferentes y en mutua oposición: la cosa pensante (res cogitans), de naturaleza espiritual (alma) y la cosa extensa (res extensa), de naturaleza corpora (Barragán L, 1997: 29). De ese modo, exalta la razón humana como facultad cognoscitiva y único medio para obtener el conocimiento válido, con la consecuente depreciación del conocimiento sensible (Gajate, 1999: 9 11). Además, supuso que "el cuerpo no era otra cosa que una estatua o máquina terrestre que Dios forma deliberadamente para hacerla lo más parecida posible a nosotros mismos" (Feher, Naddaff, Tazi, 1990: I, 460), el cuerpo es una especie de autómata (Descartes, 1977: 123) y que, si bien es cierto "tenía una cara, manos, brazos, tan solo se trataba de una maquinaria compuesta de carne y huesos $^{6}$, como se ve en un cadáver (Gajate, 1999: 9, 15) Para el filósofo francés el yo pensante no es el cuerpo, sino el alma.

El currículo en la modernidad cartesiana se caracterizó por una educación racionalista (Atkinson y Maleska, 1966: 62), absolutista en detrimento despótico de la educación física como extensión. Una educación nobiliaria con acento naturalista que orientó a los nobles aristócratas y quienes se asignaron en exclusividad la capacidad de razonar sobre el hombre común y corriente (Atkinson y Maleska, 1966: 48). Se pretendió la felicidad terrena y una "razonable" educación del cuerpo (Rodríguez, 1996: 149). Los ejercicios admitidos y propios de la sociedad cortesana eran aquellos que no presentan rudeza ni riesgo, como equitación, esgrima, danza minué y los juegos de pelota. La competencia es

\footnotetext{
6 En su pensamiento ya intuía que el cuerpo humano era una cosa entre las cosas. Por su naturaleza, lo estudió comparándolo con la mecánica del movimiento animal. (Cfr. Valserra, Fabricio. Historia del Deporte. Madrid:
} Plus Ultra, 1944, p. 222) 
mplazada por los juegos de azar. Los estudios de Desrtes sobre la mecánica animal aplicada al cuerpo huano permitieron una concepción mecanicista del moniento del hombre y el interés científico por el cuerpo mano. La supremacía del pensamiento implicó, a su la concepción del hombre-máquina y la subvaloran de la educación física nobiliaria para el entreteniento. Con su concepción dualista del hombre reforzó supremacía del pensamiento sobre el movimiento, lo al implicó la concepción una educación pensante y moviente -la una en el aula la otra en el patio-, con consecuente subvaloración mecanicista del cuerpo y educación física.

La antropología filosófica monista de Parménides y pinoza sobrevalora la espiritualidad del hombre, mienas la ciencia sobrevalora su materialidad reduciendo educación física.

Desde esta perspectiva se sostiene que hay un solo po de sustancia o realidad, bien espiritual (Parménides Spinoza) o material (ciencia).

La postura filosófica de Parménides se considera mísica debido a su presentación poética en la cual, a través e un viaje insólito desde la oscuridad de la ignorancia acia la luz de la verdad, se descubren las limitaciones -1 hombre para alcanzar ese conocimiento verdadero, ien, en consecuencia, requiere la ayuda divina, y así, s hijas del sol conducen al sendero que lleva a la dio(verdad) mediante la revelación e instrucción para el ser humano acceda al Ser (Parménides, 1965: 25). n su ruta indagatoria poética la diosa le revela que "el es y el No-ser no es" (Parménides, 1965: 53). Basado ello plantea la "teoría de los dos mundos": "el mundel no ser" (cambiante y conocido por los sentidos), "el mundo de lo que es" (absoluto y conocido por la razón).

Spinoza es considerado panteísta pues reduce todo la causa inmanente de las cosas que es la "substancia infinita", en cuyo seno se hallan los atributos con sus infnitos modos. Desde allí, plantea la "teoría de la sustancia", mediante la cual demuestra la existencia de una sola sustancia que es "Dios o Naturaleza" (Spinoza, 1958: 11). través de su método, tomado del "modo geométrico", afirmó que se debe comenzar por lo que es lógica y ontológicamente anterior, es decir la "esencia divina" y, por Heducción, continuar con todo lo demás (Gajate, 1999: . El hombre está entre "aquellas cosas que se siguen e la esencia de Dios", pero analiza las acciones y deseos umanos como si se tratara de cuerpos geométricos. El cuerpo humano es "el modo que expresa de cierto y determinado modo la esencia de Dios"(Gajate, 1999: 50).
De esa manera, el hombre es el cuerpo humano y es el alma humana en cuanto que cuerpo y alma son dos modos vectoriales de un solo ente (Spinoza, 1958); con ello soluciona el problema del dualismo.

El monismo antropológico científico-materialista defiende la materialidad del hombre en su manifestación del cuerpo humano. El conocimiento científico ha sido capaz de alcanzar el control, manipulación y transformación de la materia humana. Desde mediados del recién siglo pasado, el hombre se ha convertido en el objeto de estudio de la mayoría de las ciencias (biología, química, física, mecánica, neurología, genética y cibernética). "Todos ellos han caído en la misma fosa reduccionista osando afirmar que el hombre no es más que..." (Lobato, 1994: 96).

No se alcanza a evidenciar la supuesta influencia del monismo antropológico espiritualista en el currículo de la educación física antigua y moderna. Sin embargo, es explícitamente manifiesto en ella el cientifismo antropológico materialista. Desde Descartes el desarrollo de la ciencia ha extendido sus dominios a la explicación -y no contemplación- de la realidad del hombre. Además de racionalista, la educación fue concebida como científica; de allí su inclusión en el currículo de la educación física por el interés en enseñar la constitución del cuerpo desde las ciencias naturales.

La antropología filosófica unicista, desde el pensamiento semita, aristotélico, agustiniano, tomista, zubiriano y lainiano, emerge para reivindicar la unidad del hombre y posibilita el diseño de un currículo integral para la educación física y la cultura física.

Las tradiciones orientales nos sugieren un enfoque unitario: "espiritualidad corporal o corporalidad espiritual" (Masiá, 2002: 201). Los semitas conciben el ser humano como un todo (Dussel, 1969: 25). En la antropología hebrea, el hombre es espíritu (ruaj, pnéuma), mente ( $p$ siqué) y carne (basár, soma) en unidad ${ }^{7}$. En la Biblia es un ser esencialmente relacionado (Diccionario enciclopédico de teología moral, 1980). "Y Dios el Señor formó al hombre del polvo de la tierra, y sopló en su nariz hálito de vida, y el hombre se convirtió en un ser viviente" (Gn 2,7).

Aristóteles, desde su "teoría hilemórfica" (hylé-morphé), se aproxima a la unicidad antropológica al concebir la realidad humana (realismo) como la unión sustancial de materia y forma (Aristóteles, 1978: II, c I, $\left.412^{a}, 10,168\right)$. El Estagirita concibe al hombre ontológicamente como ser o ente y lógicamente con la dife-

\footnotetext{
7 En esta afirmación, Dussel cita obras de Tresmontant, D. Lys, A. Glein y otros.
} 
rencia específica de la racionalidad. San Agustín desde la "doctrina de la Creación, aportó la naturaleza divina del hombre con un alma de tal calidad que por la razón y el entendimiento aventaja a todos loa animales" (San Agustín, 1979: XII, 22, c, XXIV, 284), y comparte la antropología paulinocristiana respecto de la unidad espíritu-alma-cuerpo (1 Tes 5, 23). El pensamiento antropológico tomasino tiene una doble vía: una fe teológica (descendente) y una razón filosófica (ascendente) (Lobato, 1994: 37). El Aquinate sigue a Aristóteles filosóficamente y a Cristo teológicamente. El hombre es un "ser completo", no tiene cuerpo y alma, sino que "es cuerpo, alma y espíritu" (Aquino, 1, q. 75, a5, 182). El cuerpo y el alma son partes integrales y constituyentes del animal hombre (Aquino, 1977: 39-41). “El alma está toda en todo el cuerpo y toda en cada una de sus partes" (Aquino, 1998: 218). Para Tomás de Aquino, el cuerpo humano no es una realidad aislada ni yuxtapuesta a otra, sino la unión sustancial de dos principios constitutivos: el espiritual y el material (Aquino, 1, 75, 171).

Zubiri (1986: 48) sostiene que el hombre es una realidad en unidad sustantiva. -no sustancial-, un sistemaconstructo-estructural de "notas" adventicias "de sî" y constitucionales "de sustantividad" (Zubiri, 1982: 89), que conforman la totalidad sustantiva psico-orgánica. Lo psíquico es un subsistema como "nota de" todo el sistema y está constituido por algunas notas psíquicas (intelección, volición, afección). A su vez, lo orgánico es un subsistema como "nota de" todo el sistema sustantivo que es el hombre. Está conformado de notas físicoquímicas (órganos y sustancias). Además, la constitución de esa realidad humana presenta tres momentos consecutivos: organización (las notas psiquicoorgánicas se organizan tomando posición), solidaridad (las notas psicoorgánicas se solidarizan cooperando entre sí) y corporeidad (las notas se corporizan haciendo presencia en la realidad) (Zubiri, 1982: 91). Del mismo modo, dicho subsistema orgánico tiene tres funciones en el sistema total: una función organizadora (las notas fisicoquímicas contribuyen a la organización de todo el sistema), una función configuradora (las notas fisicoquímicas dan figura a todo el sistema) y una función somática (las notas fisicoquímicas materializan la corporeidad de todo el sistema) (Zubiri, 1982: 94, 97). En suma, así es posible la presencia del hombre en el mundo.

Pedro Laín Entralgo, continuando la obra de su maestro Zubiri, adiciona a la constitución y comprensión del hombre mediante su paradigma de la conducta. Además de la naturaleza psicoorgánica del ser humano, encuentra la conducta humana como realidad del hombre. Di- cha conducta es el "resultado diacrónico y global de la sucesiva actividad psicoorgánica de un sujeto personal que vive en el mundo" (Entralgo, 1989: 286). La conducta es "nota de" todo el sistema psicoorgánico, y está constituida por varias notas diferenciales: el libre albedrío (libertad para hacer lo que se quiere), la simbolización (reducción de datos sensorio-perceptibles a signos simbólicos), la visión (vivencia de las cosas como realidades), la inconclusión (modificación permanente de la acción y el ensimismamiento: recluirse en uno mismo. Plantea que esa conducta requiere de 1) algunos presupuestos: conservación de la figura bipedestante (génesis, aspecto y función), el mantenimiento de la vida (funcionalidad de los sistemas orgánicos) y la actividad sexual (mantenimiento de la especie); 2) un gobierno (cerebro); 3) una ejecución (apropiación o rechazo mediante la locomoción, expresión y excreción) (Entralgo, 1989: 311-316).

El proyecto educativo semita ha estado adherido a sus ideales religiosos y morales, que han mantenido la unidad de los judíos a pesar de su dispersión. Se enseñaba la lealtad al grupo, la lectura y escritura, estudio del Pentateuco, manteniendo fidelidad a la lengua y religión comunes. Una educación para la santidad de la conducta en obediencia y fidelidad a Dios, para asegurar la armonía civil. Los padres enseñaban un oficio y manualidades artesanas (Atkinson y Maleska, 1966: 1113). Aunque no consideraron una educación física propiamente dicha, la ley mosaica incluía algunas reglas de higiene y aconsejaba a los padres enseñar a nadar a sus hijos (Atkinson y Maleska, 1966: 14). También se sabe de sus costumbres dancísticas con sentido de adoración, gratitud y alabanza mediante las cuales todo el hombre alaba a Dios.

El contenido curricular de Aristóteles es similar al platónico, Sin embargo, su aporte más significativo es su filosofía del movimiento. Según ésta, el movimiento puede ser de cuatro clases: corrupción, crecimiento, alteración y traslación. Estos pueden tener un carácter natural o accidental. El primero consiste en el cambio natural sustancial cuyo resultado puede ser la generación de una sustancia nueva, es decir, llegar a ser ${ }^{8}$, o la destrucción $n^{9}$ de una sustancia ya existente, es decir, dejar de ser. Los tres movimientos restantes obedecen a modificaciones no esenciales de su ser. Estas pueden aparecer

8 El proceso de concepción humana podría considerarse un movimiento por generación a partir de Aristóteles.

9 El proceso corruptivo físico tras la muerte humana puede corresponder al movimiento destructivo aristotélico. 
cuantitativas si los cambios de la sustancia son de tamaho, de menor a mayor (aumento) y de mayor a menor (disminución), o cualitativas si los cambios son de la calidad como paso de una sustancia a otra (alteración), - de lugar como paso de un espacio a otro (traslación) Aristóteles, 1978: I, c, III, 406a, 10, 145). Entonces, de acuerdo con Aristóteles, si el alma es la que mueve al cuerpo, es lógico que lo mueva en concordancia con los movimientos de ella misma. Se afirma también "que el movimiento a que está sometido el cuerpo es el mismo que aquel a que está sometida el alma" (Aristóteles, 1978: I, c, III, 406b, 30, 145). Más aún, como el cuerpo experimenta el movimiento de traslación, entonces el alma se desplazará con él. De allí se sigue que el alma mueve al cuerpo y éste a aquella.

Con la aparición del Cristianismo se establece un modelo educativo basado en la moralidad y la espiritualidad. La educación cristiana se difundió rápidamente; sus métodos eran ascéticos y disciplinarios, dando más importancia a la fe que a la razón. Tendió a cubrir el cuerpo debido a la concepción de su naturaleza pecaminosa: cuerpo pecador. Los juegos y diversiones eran considerados pecaminosos, de ahí que las actividades festivas y la educación física no fueron bien vistas; en su defecto, pretendió educar el espíritu ${ }^{10}$. Con el poder en sus manos, el emperador Teodosio -convertido al cristianismo- abolió los Juegos Olímpicos (Valserra, 1944: 137) y sus carnavales a finales del siglo IV d. C. La naturaleza pagana, el alto contenido violento, sangriento e incluso mortal, el carácter orgiástico de las festividades carnavalescas y la desnudez del cuerpo humano, característica de dichas prácticas, fueron consideradas un atentado contra la moral y el Estado.

Durante la Edad Media baja, el pensamiento tomista propició una reapertura hacia las prácticas corporales su beneficio en la salud. Él mismo recomendaba los ejercicios y los juegos en la formación de los jóvenes, considerándolos necesarios para el bienestar de la vida humana.

\section{Discusión}

El dualismo antropológico platónico sigue influenciando el currículo de la educación física y cultura física con su consecuente y actual subvaloración.

La dedicación exclusiva al espíritu deduce una consideración monista, a pesar de la concepción antropológica trinitaria espíritu-alma- cuerpo, propia del pensamiento bíblico paulino, (Cf. 1, Ts 5,23).
Los actuales currículos conservan cierto dualismo platónico al considerar una educación intelectual que se desarrolla en las aulas, y una educación física que se realiza en el patio o la cancha. Aún hoy es más importante el hombre intelectual que el operario. Tienen mejor reconocimiento político, económico, social y cultural las profesiones intelectuales -entre ellas la filosofía- que las corporales como la educación física y la misma cultura física. Se mantiene aún la dualidad teoría y práctica. El currículo específico de la educación física y cultura física actuales defiende el idealismo de Platón al fomentar la búsqueda del "cuerpo ideal" saludable, fortalecido, modelado y competitivo; además, por la supuesta alma racional de los dirigentes del deporte y la recreación, concupiscible de los artistas y meramente irascible de los deportistas y recreadores. Hoy es más importante el cuerpo que el alma. También es herencia platónica la consideración de que quienes se dedican con exclusividad a la cultura física son duros y brutos, mientras que los que cultivan tan sólo el intelecto son blandos y perezosos.

El dualismo antropológico cartesiano está vigente en el currículo extensivo de la educación física con su consecuente y actual subvaloración.

Además de las influencias platónicas, los modelos pedagógicos actuales fomentan el dualismo cartesiano al proponer currículos racionales para intelectuales (ingenierías, administración, economía, ciencias políticas entre otras) y currículos extensivos para prácticos (educación física, cultura física y afines). Hoy se concibe aún el dualismo entre fe y razón, entre teoría y práctica, enseñanza y aprendizaje. Algunas propuestas curriculares de la educación física y la cultura física consideran más importante el pensar que el mover, mientras que otros mantienen ese cartesianismo al dedicarse al mecanicismo del movimiento y a las prácticas corporales sin interesarse por el pensamiento.

La influencia del monismo antropológico espiritualista de Parménides y Spinoza no se hacen evidentes en el currículo actual, mientras el materialismo cientificista continúa su influencia.

Tal vez los currículos que conservan la influencia antropológica monista espiritualista sean aquellos de corte seminarista, que preparan a novicias, monjas, frailes y sacerdotes. Pero los currículos de la educación física y cultura física, conservan explícitamente una formación fundamentada en la antropología filosófica del monismo materialista, dado su extremo interés en dar cuenta del hombre y su cuerpo a través de las ciencias naturales, en especial biología, neurología y genética. 
Es posible proponer un currículo integral y humanista desde el unicismo antropológico, considerando aspectos de sus postulados:

El currículo de la educación física y cultura física, desde una perspectiva antropofilosófica unicista, debe considerar: a) el diseño, desarrollo y evaluación en unidad coherente; b) la unidad psico-orgánico-conductual del hombre (estudiante); c) la unidad curricular formativa científico-humanista-cultural; d) una gestión curricular sustantiva (pertenencia social y pertinencia académica, evaluación formativa, interdisciplinariedad, iinvestigación e innovación, elaboración permanente y colectiva, participación, flexibilidad y practicidad); e) un comité curricular interdisciplinario; f) una comunidad académica sustantiva interdisciplinaria, comunitaria e investigativa; g) una docencia sustantiva (academia, capacitación, administración, investigación, comunidad y renovación de saberes); h) la determinación de unidades académicas curriculares para cada semestre o ciclo de formación integrando núcleos temáticos, problemáticos y programáticos - no asignaturas sino disciplinas-, considerando la unidad teórico-práctica.

\section{Conclusiones}

- Las antropologías filosóficas dualistas y monistas no satisfacen la comprensión y educación humanas.
- La antropología filosófica unicista favorece la concepción unitaria y actual del currículo de la educación física.

- Se requiere mayor formación humanista en los currículos de la educación física y cultura física.

- Es necesario reconsiderar el fundamento epistemológico de los términos educación física y cultura física, dado que implican aparentemente una concepción monista materialista actualmente cuestionada.

- La docencia debe ir más allá de la academia magistral y la dictadura de clase.

- Debe desaparecer la concepción dualista de las "clases teóricas" y las "clases prácticas", pues la teoría es práctica y la práctica es teórica.

- Debe asumirse que el alma es corporal y el cuerpo anímico incluso espiritual, "lo intelectual es práctico" y "lo práctico es intelectual", la cultura física es intelectual y la cultura intelectual es física. Se debe reconocer que el cuerpo humano es más que un instrumento, materia o máquina.

- El encargo social de la educación física es precisamente el bienestar de la sociedad.

- Finalmente, sólo con estudio e investigación rigurosa la educación física y la cultura física pueden ser reconocidas en el ámbito de la comunidad académica y científica. (D)

\section{Referencias}

Aquino, Tomás de. Suma Teológica: Tratado del hombre.

Aquino, Tomás de. Del ente y la esencia. Buenos Aires: Aguilar, 1977. Aquino, Tomás de. Suma contra los gentiles. México: Porrúa, 1998.

Aristóteles. Sobre el alma. Madrid. Gredos. 1978.

Atkinson y Maleska. Historia de la Educación: Barcelona. Martínez Roca, 1966.

Barragán L., Hernando. Filosofía Moderna. Bogotá: Universidad Santo Tomás de Aquino, 1997.

Descartes, René. Discurso del Método. México: Porrúa, 2004a.

Descartes, René. Meditaciones metafísicas. México: Porrúa, $2004 \mathrm{~b}$.

Descartes, René. Meditaciones metafísicas. Madrid: Alfaguara, 1977.

Diccionario enciclopédico de teología moral. Madrid: Paulinas, 1980.

Dussel, Enrique. Humanismo semita. Buenos Aires: Eudeba, 1969.

Entralgo, Laín, P. El cuerpo humano: Teoría actual. España: Espasa Calpe. 1989.

Feher, Michael, Naddaff, Ramona y Tazi, Nadia, citando a Descartes. Fragmentos para una historia del cuerpo humano. Madrid: Taurus, 1990.
Gajate M., José. Historia de la filosofía. Bogotá: El Búho.1999.

Jaeger, Werner. Paideia. Bogotá: Fondo de Cultura Económica. 1997. Lobato, Abelardo, O. P. y otros. El hombre en cuerpo y alma. España. Edicep C. B., 1994.

Masiá C., Juan. Caminos sapienciales de oriente. Bilbao: Desclée de Brouwer. 2002.

Parménides. Fragmentos: Sobre la naturaleza. Buenos Aires: Aguilar, 1965.

Platón. Diálogos: Fedón o del alma. México: Porrúa. 2003.

Rodríguez A., Eudoro y otros. Antropología: perspectiva latinoamericana. Bogotá, Universidad Santo Tomás de Aquino, 1996.

Spinoza, Baruch. Ética: demostrada según el orden geométrico. México: Fondo de Cultura Económica, 1958.

San Agustín. La ciudad de Dios. México: Porrúa. 1979.

Valserra, Fabricio. Historia del Deporte. Madrid: Plus Ultra, 1944.

Zubiri, Xavier. Sobre el hombre. Madrid: Alianza, 1986.

Zubiri, Xavier. Siete ensayos de antropología filosófica. Bogotá: Universidad Santo Tomás de Aquino, 1982. 